\title{
Tumores testiculares y paratesticulares prepuberales. Experiencia en nuestro centro y revisión de la literatura
}

\author{
E. Cao Avellaneda, H. Alarcón Martínez*, J.L. Fuster Soler**, P. López Cubillana, \\ E. Llinares Riestra**, M. Pérez Albacete
}

Servicio de Urología. *Servicio de Pediatría. ** Unidad de Oncología Infantil. Hospital Univ. Virgen de Arrixaca. Murcia.

Actas Urol Esp 2005; 29 (4): 355-359

\begin{abstract}
RESUMEN
TUMORES TESTICULARES Y PARATESTICULARES PREPUBERALES. EXPERIENCIA EN NUESTRO CENTRO Y REVISIÓN DE LA LITERATURA

Objetivos: evaluar la importancia de los tumores testiculares y paratesticulares en nuestro medio, así como realizar una puesta al día sobre el tema.

Métodos y pacientes: se ha revisado la base de datos de la Unidad de Oncología del hospital Universitario "Virgen de la Arrixaca" desde el 1 de Enero de 1998 hasta el 31 de diciembre del 2003, extrayendo los casos referidos a neoplasias en esta localización.

Resultados: se han detectado 7 neoplasias entre los 190 pacientes tratados (representa el 3,68\% del total de tumores): 5 testiculares y 2 paratesticulares. Las estirpes testiculares fueron: un tumor del seno endodérmico, un teratoma maduro, un tumor de células de Sertoli, uno de tipo estromal mixto o indiferenciado y un caso de linfoma de Burkitt. Los dos tumores paratesticulares fueron rabdomiosarcomas. La actitud inicial fue orquiectomía radical en todos los casos, salvo una escrotectomía parcial en un caso de rabdomiosarcoma debido a cirugía transescrotal previa y quimioterapia aislada en el caso del linfoma. Los rabdomiosarcomas requirieron quimioterapia adyuvante. Todos los pacientes están vivos y libres de recidiva con un seguimiento entre 17 y 74 meses.

Conclusiones: los tumores testiculares y paratesticulares son infrecuentes en nuestra práctica habitual. Salvo en un caso de linfoma, el tratamiento quirúrgico inicial ha sido esencial en su manejo. En nuestra serie tienen un excelente pronóstico.
\end{abstract}

Palabras clave: Tumores testiculares. Tumores paratesticulares. Infancia.

\section{ABSTRACT \\ TESTICULAR AND PARATESTICULAR PREPUBERAL TUMORS: OUR EXPERIENCE AND UPDATE ON THE TOPIC}

Objetives: to evaluate the importance of testicular and paratesticular prepubertal tumors in our center and to make an update on the topic.

Methods and patients: data from all patients diagnosed of testicular and paratesticular prepubertal tumors and treated in our pediatric oncology unit from January $1^{\text {st }} 1998$ to December $31^{\text {st }} 2003$ have been revised.

Results: seven cases are reported among one hundred and ninety patients (represents 3,68 percent of all treated tumors): five tumors affecting the testis and two cases of paratesticular tumors. Pathology classification was as follows: one yolk salk tumor, one mature teratoma, two nongerminomatous testicular tumors (one Sertoli cell tumor and one unclassifiable), one Burkitt's lymphoma and two paratesticular rhabdomyosarcomas. Primary approach was inguinal radical orchiectomy in all cases except neoadjuvant chemotherapy in the case of lymphoma and partial escrotectomy in one patient previously managed with transcrotal orchiectomy. Rhabdomyosarcoma cases received adjuvant chemotherapy. All patients are alive and well after a follow-up period ranging from 17 to 74 months.

Conclusions: testicular and paratesticular prepubertal tumors are rare. Except for one patient affected of lymphoma, surgical primary approach have been essential for treatment. The prognoses in this series has been excellent.

Keywords: Testicular tumors. Paratesticular tumors. Childhood. 
$L^{2}$ os tumores testiculares y paratesticulares en la edad prepuberal tienen notables diferencias en su distribución y comportamiento respecto a periodos posteriores, por lo que requieren un abordaje independiente. A nivel nacional, se han publicado numerosas series de casos clínicos, siendo éstas referidas mayoritariamente a una única estirpe. El estudio retrospectivo de las mismas sobre el global de neoplasias en un periodo de 5 años cobra especial importancia para conocer tanto su frecuencia relativa como la propia distribución histopatológica dentro del grupo.

\section{PACIENTES Y MÉTODOS}

Se han revisado los archivos clínicos de la Unidad de Oncología Pediátrica del Hospital "Virgen de la Arrixaca" entre el 1 de Enero de 1998 y el 31 de Diciembre del 2003, extrayendo el total de neoplasias tratadas en el Servicio, asî como las de localización testicular y paratesticular. En estos casos, se ha revisado la historia clínica. Se expone la edad, estirpe histológica, estadio clínico-patológico, marcadores, tratamiento recibido, seguimiento y estado actual. Posteriormente, se realiza una puesta al día de los aspectos más destacados en este tipo de neoplasias, tras la revisión de la literatura mediante el uso de artículos y resúmenes obtenidos en la base de datos Pubmed sin limitación de fecha de publicación.

\section{RESULTADOS}

Entre un total de 190 pacientes tratados por neoplasia de órgano sólido en nuestro hospital se han detectado un total de 7 tumores: 5 de localización testicular y 2 paratesticular. Esto representa el 3,68 \% del total de tumores tratados. La mediana de edad al diagnóstico es 22 meses con un intervalo entre catorce meses y 132 meses. Las estirpes histológicas encontradas fueron: dos rabdomiosarcomas paratesticulares, un tumor del seno endodérmico, un tumor de las células de Sertoli, un tumor estromal inclasificable, un teratoma maduro y un linfoma de Burkitt. El estadio diagnosticado fue localizado (estadio I) en todos los casos, salvo en el linfoma de Burkitt, que correspondía a estadio III. Determinamos niveles elevados de alfa-fetoproteína sólo en el caso del tumor del seno endodérmico, sin haberse realiza- do su determinación en los casos de rabdomiosarcoma y en el caso de linfoma. El tratamiento recibido fue orquiectomía radical aislada para los casos de tumor del seno endodérmico, tumor de células de Sertoli, tumor estromal inclasificable y teratoma maduro. Todos los tumores citados están libres de recidiva con un seguimiento de 29, 47, 17 y 24 meses respectivamente. Ambos rabdomiosarcomas fueron tratados inicialmente con cirugía; el primero de los casos se sometió a escrotectomía parcial debido a un abordaje inicial incorrecto vía escrotal, mientras el segundo caso se sometió a orquiectomía radical. La quimioterapia para el primer rabdomiosarcoma fue la rama 952 del protocolo MMT-95 (malignant mesenchymal tumors) de la Sociedad Internacional de Oncología Pediátrica (SIOP), más enérgica que la recibida en el segundo caso, la rama 951 del mismo protocolo. Finalmente, el caso de linfoma de Burkitt diagnosticado por masa escrotal fue sometido a quimioterapia según el protocolo de la Sociedad de Hematología y Oncología Pediátrica (SHOP-94). Tanto los casos de rabdomiosarcoma, con 74 y 44 meses de seguimiento respectivamente, como el caso de linfoma con 72 meses están libres de recidiva. Por lo tanto, la supervivencia global en nuestra serie es del 100\%, con una media de seguimiento de 56,3 meses (intervalo entre 17 y 74 meses).

\section{DISCUSIÓN}

La distribución anatomo-patológica de los tumores testiculares prepuberales difiere considerablemente de la encontrada en el adulto. En un estudio epidemiológico europeo, Weissbach analizó la distribución de los tumores testiculares en adultos y en niños. En los primeros, el 89\% estaba constituído por seminomas (lo más frecuente), carcinoma embrionario y tumores mixtos. En los niños, el tumor del saco endodérmico y el teratoma son los más frecuentes, con un $62 \%$ del total entre ambos ${ }^{1}$. El 69\% de los tumores testiculares en adultos se encuentran extendidos en el momento del diagnóstico, hecho que sólo se observa en un $9 \%$ de los casos diagnosticados en niños.

En este período prepuberal, los sarcomas representan entre el 5 y el 15\% de todas las neoplasias, siendo el rabdomiosarcoma el más fre- 
cuente $^{1,2}$. Otro hecho diferencial es la alta proporción de histologías benignas diagnosticadas, hasta un $30 \%$ en el registro de tumores testiculares prepuberales de la asociación americana de Urología $^{1-3}$. Respecto a los resultados de nuestra serie, es dificil, por lo limitado de la misma, establecer siquiera comparaciones de semejanza. Destacar la aparición de los tres tumores más frecuentes en la literatura revisada: el tumor del saco vitelino, el teratoma y el rabdomiosarcoma.

El motivo de consulta más frecuente es la masa testicular. Se asocian hasta en un $20 \%$ de los casos a hidroceles reactivos y hasta en un $21 \%$ a hernias inguinales ${ }^{2-4}$.

Es fundamental el estudio de algunos marcadores tumorales como la alfafetoproteína (AFP) y la fracción beta de la gonadotropina coriónica humana ( $\beta-H C G)$ previos a la cirugía. En algunos protocolos norteamericanos, la negatividad de ambos es criterio indispensable para la posible cirugía conservadora $^{2,5}$. El estudio de extensión de debe realizar mediante pruebas de imagen, que en ningún momento deben retrasar la realización del abordaje quirúrgico; el factor fundamental para la decisión terapéutica final es el estudio histológico de la pieza ${ }^{5,6}$.

Se recomienda la utilización del estadiaje según el Pediatric Oncology Group (POG) y el Children's Cancer Group (CCG) ${ }^{9}$ (Tabla 1). Los criterios de inclusión en el estadio I (localizado) además de la verificación del foco tumoral limitado al testículo, requieren la ausencia de diseminación macroscópica durante la cirugía y la negatividad de pruebas de imagen y marcadores tumorales previos y tras las dos primeras semanas postcirugía.

$\mathrm{El}$ abordaje quirúrgico es por vía inguinal. Se debe realizar con clampaje atraumático de cordón inicial para exploración y toma de muestra previa si procede. En caso de necesidad de cirugía radical, se realiza la excisión en bloque de las estructuras asociadas del cordón espermático y testículo. Los procedimientos quirúrgicos que violen el escroto, las túnicas vaginales y la albuginea se asocian a mayor riesgo de recidiva inguinal y retroperitoneal, por lo que están contraindi$\operatorname{cadas}^{7,8}$.

La realización de cirugía conservadora como opción quirúrgica inicial fue comunicada por pri-
Tabla 1

Estadiaje según el Pediatric Oncology Group (POG) y el Children`s Cancer Group (CCG).

\begin{tabular}{cl}
\hline ESTADIO & EXTENSIÓN DEL TUMOR \\
I & - Limitado a testículo. \\
& - Resección completa tumoral sin \\
& diseminación. \\
& - No evidencia radiológica y patológica de \\
& extensión. \\
& - Marcadores (-) ó biopsia retroperitoneal (-). \\
& - Diseminación macroscópica del tumor \\
& en cirugía. \\
II & Enfermedad microscópica a menos de \\
& 5 cm del extremo proximal del cordón o \\
& escroto afecto. \\
& - Adenopatías retroperitoneales menores \\
& de 2 cm. \\
& - Aumento de marcadores tras disminución \\
& inicial. \\
\hline III & - Adenopatías retroperitoneales mayores \\
& de 5 cm. \\
\hline IV & - Metástasis a distancia. \\
\hline
\end{tabular}

mera vez a mediados de los años 80 y se generalizó en la siguiente década, destacando los trabajos de Sugita ${ }^{10}$. El CCG/POG recomienda el inicial abordaje exploratorio inguinal. Son candidatos a la cirugía conservadora aquellos que reúnen los siguientes criterios:

1. negatividad de AFP preoperatorio para los pacientes mayores de 1 año. Entre los 6 a 12 meses deben de estar por debajo de 100 $\mathrm{ng} / \mathrm{ml}$.

2. testículo sano potencialmente respetable por ecografía.

Si el paciente cumple estos criterios, tras el inicial clampaje atraumático del cordón, se realiza biopsia escisional de la masa. Si se obtiene confirmación histológica de benignidad tumoral con límites quirúrgicos libres, se procede al cierre del teste restante. En caso de histología maligna o duda sobre su posibilidad, se procederá a realizar orquiectomía radical. En el caso particular de pacientes cerca de la pubertad y diagnóstico de teratoma, se requiere además la confirmación del estado prepuberal del tejido sano adyacente para su realización ${ }^{2}$.

El tratamiento específico dependerá de cada estirpe histológica y su estadio clínico-histológico. A los pacientes tratados en nuestro hospital no se realizó en ninguno de los casos cirugía 
conservadora debido a la escasa edad de casi todos ellos, circunstancia que dificulta esta actitud, por la mayoría de estirpes potencialmente malignas encontradas y la poca experiencia de nuestro centro (Tabla 2).

El tumor del saco vitelino es el más frecuente en la edad prepuberal. Se diferencian de los del adulto en la casi total ausencia en los mismos de formas mixtas. En el 85\% de los casos se diagnostican en estadio I, con una tasa global de supervivencia de más del 90\%. Dos terceras partes de los mismos se curan tras la orquiectomía radical. En estadios II y III se recomienda la quimioterapia inicial con posterior cirugía citorreductora y/o linfadenectomía retroperitoneal posterior según los $\operatorname{casos}^{11-13}$.

El teratoma en la edad prepuberal representa entre 10 al $25 \%$ del total de estos tumores, diagnosticándose fundamentalmente antes de los 4 años de edad. Su comportamiento en los niños suele ser benigno, incluso con la presencia de elementos histológicos indiferenciados. Por ello, se puede realizar cirugía preservadora de testículo sano, con el consecuente beneficio funcional y psicológico del paciente, aunque esta actitud no está unánimemente aceptada. En casos limítrofes por la edad, se requiere la verificación anatomopatológica del estadio prepuberal previo a la cirugía conservadora ${ }^{14-16}$.

El rabdomiosarcoma paratesticular es el tercer tumor en frecuencia. Por la complejidad de su manejo, requiere un tratamiento individulizado y multidisciplinario. La SIOP recomienda la realización de orquiectomía radical vía inguinal. Como ya hemos referido anteriormente, se debe realizar escrotectomía parcial en el caso de abordaje transescrotal previo. El tratamiento adyuvante se realiza siguiendo los protocolos de dicha organización, dependiendo del análisis anatomopatológico de la pieza y de estudios de extensión ${ }^{17,18}$.

$\mathrm{El}$ quiste epidermoide supone el cuarto tumor en frecuencia con un $3 \%$. Se define por estar formado en su totalidad por epitelio productor de queratina. Se debe diferenciar del quiste dermoide, que contiene otros anejos cutáneos, y del teratoma, que incluye la tres capas embrionarias (endo-/meso- y ectodermo). Así como el teratoma, el quiste epidermoide prepuberal puede ser considerado un tumor benigno. La realización de cirugía conservadora testicular es una opción a considerar ${ }^{14}$.

Los neoplasias estromales del testículo engloban a los tumores de células de Leydig, de células de Sertoli, a los tumores de la granulosa juvenil y a los mixtos o indiferenciados, que en su conjunto suman un total del 10\%. El tumor de las células de Leydig aparecen entre los 5 y 10 años de vida. Su diagnóstico es precoz por la aparición de signos clínicos de pubertad. Son universalmente considerados benignos en esta edad. Se puede plantear la cirugía conservadora, aunque la indicación no es tan clara como en el teratoma y quiste epidermoide ${ }^{19}$.

El tumor de las células de Sertoli suele detectarse en periodos precoces de vida. Aunque mayoritariamente benignos en su evolución, se han comunicado casos de afectación metastásica, por lo que está indicada la orquiectomía radical, que suele ser suficiente en la mayoría de los $\operatorname{casos}^{20}$.

Tabla 2

Cuadro - Resumen casos clínicos

\begin{tabular}{|c|c|c|c|c|c|c|c|}
\hline & Caso 1 & Caso 2 & Caso 3 & Caso 4 & Caso 5 & Caso 6 & Caso 7 \\
\hline $\begin{array}{l}\text { Edad } \\
\text { (al diagnóstico) }\end{array}$ & 9 años & 11 años & 21 meses & 6 años y 3 meses & 22 meses & 22 meses & 14 meses \\
\hline Histología & $\begin{array}{l}\text { Teratoma } \\
\text { maduro }\end{array}$ & $\begin{array}{l}\text { Linfoma } \\
\text { Birkitt }\end{array}$ & $\begin{array}{c}\text { Rarbomiosarcoma } \\
\text { embrionario }\end{array}$ & $\begin{array}{l}\text { Rabdomiosarcoma } \\
\text { embrionario }\end{array}$ & $\begin{array}{l}\text { Tumor del seno } \\
\text { endodérmico }\end{array}$ & $\begin{array}{l}\text { TM Células } \\
\text { Sertoli }\end{array}$ & $\begin{array}{l}\text { Tumor estromal } \\
\text { inclasificable }\end{array}$ \\
\hline Estadio & I & III & I & I & I & I & I \\
\hline Tratamiento & Orquiectomía & SHOP-94 & $\begin{array}{c}1^{\circ} \text { Tumorectomía } \\
2^{\circ} \text { Escrotectomía } \\
3^{\text {o }} \text { SIOP-MMT-95 (R952) }\end{array}$ & $\begin{array}{l}\text { 1 Orquiectomía } \\
2^{\circ} \text { SIOP-MMT-95 } \\
\text { (R-951) }\end{array}$ & Orquiectomía & Orquiectomía & Orquiectomía \\
\hline $\begin{array}{l}\text { Seguimiento } \\
\text { (libre } \\
\text { de enfermedad) }\end{array}$ & 24 meses & 72 meses & 74 meses & 44 meses & 29 meses & 47 meses & 17 meses \\
\hline
\end{tabular}


El tumor de la granulosa infantil tiene un origen incierto. Se diagnostica casi exclusivamente durante el primer año de la vida. Es frecuente la alteración del cromosoma Y y/o mosaicismo en los pacientes diagnosticados. Tiene como tratamiento de primera elección la orquiectomía radical, generalmente curativa $^{21,22}$.

Finalmente, dentro de los tumores estromales encontramos los tumores mixtos e indiferenciados. No existe en la literatura suficientes pruebas como para poder establecer guías de práctica o recomendaciones respecto a él. La orquiectomía radical es su tratamiento adecuado y suficiente, aunque por lo incierto de su comportamiento se recomienda seguimiento posterior de estos pacientes ${ }^{23}$.

Los gonadoblastomas sólo representan el 1\% de los tumores testiculares prepuberales. Casi exclusivamente aparece asociado a disgenesia gonadal. Contienen tanto elementos germinales como estromales; por lo que distinguimos en ellos tres componentes: células germinales (que recuerdan al seminoma), células de los cordones y elementos estromales. El 40\% de los gonadoblastomas son asintomáticos. El crecimiento de los componentes germinales degenera en un 50\% de los casos en neoplasia, generalmente disgerminomas. El tratamiento es la orquiectomía radical. En caso de presencia del cromosoma Y, está indicada su realización profiláctica precoz. En los casos de pérdida del mismo (p.e. en sindrome Turner), es menos probable su aparición. Si existe afectación metastásica, la radioterapia adyuvante es primera línea de tratamiento posterior $^{24}$.

\section{CONCLUSIONES}

Los tumores testiculares y paratesticulares son infrecuentes en nuestra práctica habitual. El tratamiento quirúrgico inicial ha sido esencial en todos los casos, salvo en el paciente afecto de linfoma. En nuestra serie tienen un excelente pronóstico.

\section{REFERENCIAS}

1. Weissbach L, Altwein JE, Syiens R. Germinal testicular tumors in childhood. Eur Urol 1984;10:73.

2. Ross JH, Rybicki L, Kay R. Clinical behaviour and contemporany management algorithm for prepuberal testis tumors: a summary of the prepuberal testis tumor registry. J Urol. 2002; 168:1675-1679.

3. Kay R. Prepuberal testicular tumor registry. J Urol 1993; 150:671-676.
4. Coppes MJ, Rackley R, Kay R. Primary testicular and paratesticular tumors of childhood. Med Pediatr Oncol 1994; 22:329-33.

5. Cushing B, Perlman E. y cols. Germ cell tumors. En Pizzo P y Poplack D (eds). Principles and practice of pediatric Oncology. $4^{\circ}$ edición. Philadelphia. Lippincott, Williams and Wilkins publishers. 2001.

6. Pintor I, Aransay A. Tumores testiculares prepuberales. En Tratado de Oncología Urológica. Resel L y Moreno J editores. Sanidad y Ediciones Sl. Madrid. 2003.

7. Rushton HG, Belman AB, Sesterhenn E y cols. Testicular sparing surgery for prepuberal teratoma of the testis: a clinical and pathological study. J Urol 1990;144:726-30.

8. Ross JH, Kay R y Elder J. Testis sparing surgery for pediatric epidermoid cysts of the testis. J Urol 1993;144:353357.

9. Cushing R. Staging of testicular, ovarian and extragonadal tumors. J Clin Oncol 2004;22(13):2691-2700.

10. Sugita Y, Belman AB, Sesterhenn y cols. Testicular y paratesticular tumors in children:30 years'experience. Aust N J Surg. 1999;69:1999.

11. Grady RW. Current management of prepuberal yolk sac tumors of the testis. Urol Clin North Am 2000;27(3):503-508.

12. Griffin GC, Raney RB, Snyder H y cols. Yolk sac carcinoma of the testis in children. J Urol 1987; 137: 954-957.

13. Connoly JA, Gearhart JP. Management of yolk sac tumors in children. Urol Clin North Am 1993;20:7-13

14. Walsh C, Rusthon H. Diagnosis and management of teratomas ans epidermoid cysts. Urol Clin North Am 2000;27 (3):509-518.

15. Rabbani F, Farivar-Mohseni H y Leon A. Clinical outcome alter rertroperitoneal lymphadenectomy of patients with pure testicular teratoma. Urology 2003;62(6):1.092-1.096.

16. Rushton HG, Belman AB, Sesterhenn E y cols. Testicular sparing surgery for prepuberal teratoma of the testis: a clinical and pathological study. J Urol 1990;144:726-728.

17. Crist WM, Anderson JR, Meza JL y cols. Intergroup rhabdomiosarcoma study-IV: result for patients with nonmetastasic disease. J Clin Oncol 2001;198(12):3.091-3.102.

18. Kaefer M, Rink RC. Genitourinary rhabdomyosarcoma. Treatment options. Urol Clin North Am 2000;27(3):471487.

19. Merlini E, Seymandi PL, Betta PG, Bussi G. Testis sparing enucleation of a Leydig-cell tumour in a boy. Pediatr Med Chir 2003; Jan-Feb;25(1):63-65.

20. Young RH, Koelliker DD, Scully RE. Sertoli cell tumors of the testis, not otherwise specified: a clinicopathologic analysis of 60 cases. Am J Surg Pathol 1994;22:709.

21. Fagin R, Berbescu E, Landis S y cols. Juvenile granulosa cell tumor of the testis. Urology 2003;62(2):351.

22. Goswitz JJ, Pettinatto G, Manivel JC. Testicular sex cordstromal tumors in children: clinicopathologic study of sixteen children with review of the literature. Pediatr Pathol Lab Med 1996;16:451-470.

23. Ramani P, Yeung CK, HAbeebu SSM. Testicular intratubular germ cell neoplasia in children and adolescent with intersex. Am J Surg Pathol.1993;17:1124.

24. Savage MO, LoweDG. Gonadal neoplasia and abnormal sexual diferentiation. Clin Endocrinol 1990;32:519.

Dr. E. Cao Avellaneda

Jiménez de la Espada, 40 - 4º A

30203 Cartagena (Murcia)

(Trabajo recibido el 10 noviembre de 2004) 